\title{
EDITORIAL
}

\section{COVID 19- Tips for getting back to work}

\section{Anirudh Kohli}

Head of Imaging, Breach Candy Hospital Trust, 60 Bhulabhai Desai Road, Mumbai, Maharashtra, India.

E-mail: dranirudhkohli@gmail.com

We are seeing the most unbelievable and unprecedented time of our lives accompanied with significant uncertainty as to what the future has in store for us. As lockdowns are eased, it's time to get back to work! The pandemic is no way over, hence the risk of transmission and contraction of infection continues to remain significant. In Mumbai, we got it first and hard. Many parts of the country are yet to get hit! Sincerely hope they are spared, but it is better to be prepared.

Severe Acute Respiratory Syndrome Coronavirus-2 is the virus which causes COVID-19. This is a highly infectious disease which spreads human to human via droplets, aerosols and fomites. Materials which may carry infection on their surfaces are known as fomites, this is a new word for many. The virus may survive on different surfaces for different periods of time, cardboard 24 hours, stainless steel 48 hours and plastic 72 hours. ${ }^{[1]}$

Wherever there are regions of high human contact such as workplaces, social gatherings, entertainment zones, shopping areas, airports and during transportation, transmission may occur from human to human. The only way to attempt to control the spread is to break the human to human contact chain. This is the reason why lockdowns have been instituted, so that there is minimal human to human contact. However, for survival and combatting the infection, the high human contact zones during lockdown are healthcare facilities, supermarkets and pharmacies. Healthcare establishments are particularly hotspots for infection of uninfected patients as well as healthcare professionals. The imaging department is a critical component of any health care facility thus a high human contact zone. There is need for an SOP for imaging departments to limit transmission of disease from infected patients to healthcare workers, healthcare workers to other healthcare workers and infected healthcare workers to uninfected patients. We must not allow the imaging department to become a hotspot, and consequently if it becomes a containment zone, will be a disaster for any healthcare facility.

There are four main aspects in attempting to control transmission of disease in a healthcare facility. These are social distancing, personal protective equipment, hygiene and surface decontamination.

\section{Social Distancing}

To achieve social distancing in an imaging department, it is very important to reduce the number of human beings in the department by controlling the traffic and crowding in the department. These measures can be, both on the patient side as well as departmental staff side. Number of patients can be reduced by scheduling appointments so that there is no waiting and crowding in the department. Elective procedures such as mammography, DEXA, lung cancer screening, ILD, follow up studies etc. should be postponed as much as possible. A decision to scan patient should be based on how imaging will impact patient care. A discussion with the clinician often helps to decide whether imaging should be performed or not. In the past, we actively marketed imaging and welcomed a crowded imaging department. In this pandemic, we need to do the opposite which is actually de market imaging so that the imaging department is not a crowded zone. Only one relative per patient should be allowed. Since the number of patients and relatives will be reduced, number of waiting room chairs should be reduced by $50 \%$. This also assures social distancing in the waiting areas. On the departmental staff side, to achieve social distancing, only healthcare workers necessary for primary care are retained on the premises. The rest are encouraged to work from home. Front and back office staff can work from home - reports can be transcribed, appointments given, patients' queries and patient preparation can all be done by calls diverted to the secretarial staff at home. Only skeleton staff to cater to reduced load of patients is maintained. PACS is easily available, very affordable with high data transfer rates available, imaging specialists can report from home. A dream come true for many! In addition, imaging specialists can use voice recognition dictation software like Augnito and Dragon to dictate their reports. These are nearly 100 percent accurate with no learning curve, no training required and not accent dependent. Consequently, there is no need for transcriptionists. Thanks to Augnito, we have dispensed with transcriptionists, a cost saving. Staff on the premises needs to be divided into teams, consisting of all essential staff, paramedical staff, technicians, residents and consultants. These teams should work in different shifts on different days. They should not meet and interact. The idea being in case one team is exposed to a Covid positive patient and needs to be quarantined the other 
team can take over. If this is not done the department can be crippled with a large number of staff being sent into quarantine. It is also important to separate staff into two groups - those who are in direct contact and those who are in indirect contact with Covid positive patients. This will help reduce cross infection. They should not be allowed to mix, also one should try and create zones such as clean zone, buffer zone and a contaminated zone. Those coming from contaminated zones should be disinfected in the buffer zone before entering the clean zone. A hotspot which is totally against social distancing is the lunch room where staff tend to gossip, share lunch boxes, as a result of which lunch room should be monitored well, the timings of lunch staggered, reduce the number of users at a time. Above all, very important to educate the staff.

Screening patients to weed out potential infectors is useful, but far from successful as nearly $70 \%$ are asymptomatic. Prior to entry into the department as well as prior to giving an appointment the patient should be questioned for signs and symptoms of fever, sore throat, dry cough, diarrhea, headache, loss of taste, smell. Temperature checks may be done on entry to department but as $70 \%$ may be asymptomatic carriers, this has limited value.

\section{Personal Protective Equipment}

These are the most visible aspect of protection against COVID-19. Of great value when safe distance cannot be maintained between health care worker and COVID positive/suspect. These consists of Mask, gown, gloves, face, eye protection, head and shoe covers. Depending on the type of exposure whether aerosol generating or not the level of PPE required is used.

Masks: These are the most commonly used PPE. A discussion on types of masks is very important, as there have been several confusing, contradictory, inconsistent, mixed messages with frequent flip-flops from the authorities, both international as well as national. This has essentially been based on a short supply of masks and a desire to ration their use. Masks are important as asymptomatic COVID patients constitute seventy percent of cases as well as symptomatic patients start shedding the virus for a few days, before they are symptomatic.

There are three types of masks - cloth masks, surgical masks and respirators. Cloth masks are the simplest and cheapest as they can be homemade or mass produced. They are useful for non-healthcare workers, uninfected patients. These are useful in public places where it is difficult to maintain six feet distance such as grocery stores, pharmacies. However, cloth masks have no significant filtration capabilities as they are 50 times less effective than an N95 mask.

Surgical masks are triple layered where the innermost layer is of absorbent material, absorbs moisture from the wearer's breath to prevent getting wet easily, the middle layer is the important layer which consists of melt-blown material and acts as a filter, the outer layer repels liquids. The surgical masks has pleats to increase the surface area, so nose, mouth, chin are covered. The utility of surgical masks is to protect a patient during a procedure. If surgeon coughs or sneezes, patient should not get infected. Surgical masks also protect the surgeon from splashes of fluid such as blood, large particle droplets. Surgical masks are not designed to protect the surgeon from infection. It actually has a loose fit and allows air to enter from the sides, therefore does not provide any protection from airborne infections.

Respirator masks are utilized to prevent inhalation of air borne infectious material, vapors, dusts, smoke. They have a high filtration frequency to airborne particles. These are classified based on the filtration capabilities as well as type of substance filtered. They are also classified according to American and European standards. The American standard is based on the substance filtered, they are classified into $\mathrm{N}, \mathrm{P}$ and $\mathrm{R}$ types. $\mathrm{N}$ stands for when non-oily particles are filtered; $\mathrm{P}$ stands when oily particles are filtered such as with exhaust fumes; $\mathrm{R}$ is when some oily particles are filtered. These are further classified based on their filtration efficiency, percentage of particles above 0.3 microns filtered, so 95,99 and $100 \%$. N95 can filter $95 \%$ of particles greater than 0.3 micron. Similarly, the European standards define these as Filtering face piece respirators (FFP). These are classified as FFP1 FFP2, FFP3 based on their level of filtration as well as leakage along the sides. FFP1 is useful for dust, FFP2 for influenza group of viruses, FFP3 for asbestos. N95 has become a synonymous name for both American and European standard masks. These masks have two characteristics, an air filtration system to prevent passage of microorganisms and noxious substances. To achieve this millions of polypropylene microfibers are layered on top of each other. These are then electrostatically charged to maintain their ability to filter microorganisms or micro particles. The second characteristic is the tight fit. It creates a facial seal so as not to allow any air to seep in from the sides.

Two new words that we are now getting familiar with is donning and doffing. Donning is the wearing of personal protective equipment, whereas doffing is the removal of the personal protective equipment. Donning and doffing a mask is of great importance as incorrect donning may not protect the health care worker as well as give a false sense of security. Incorrect doffing may result in contact with the outer potentially contaminated surface resulting in transmission of infection.

The procedure for donning of a facemask will start with hand hygiene, place the mask on the face, covering the nose and mouth, secure the elastic bands, upper one on the crown of the head, the lower one below the ear at the base. Next is to fit and mold the flexible nose clip. Finally 
ensure it fits snuggly around the nose, face, chin with an adequate seal [Figure 1].

One of the important characteristics of an N95 mask is it should have a tight fit. To have a tight fit, sizing is very important for a proper fit and therefore the masks come in different sizes small, small-medium, medium, medium-large and large. Also, once the N95 is worn, it is important to see that there is user seal check to see that the respirator is donned correctly and this must be done every time the respirator is donned. This will ensure that there is a tight seal. To test the seal, a positive seal check is done when on exhalation the face piece should bulge and a negative seal check is done on inspiration the face piece should collapse. It is very important that if an individual has a beard one should shave the beard as the tight fit is not possible with a beard.

The doffing again starts with hand hygiene. It is very important not to contact the front surface of the masks, because this may be potentially contaminated. To avoid this, grasp the bottom elastic, bring it forward over your head, then with the upper strap over the head and remove the mask holding both ties so the masks is not touched at all. Dispose the mask off as infectious waste [Figure 2].

There are numerous controversies and queries regarding N95 masks. These are regarding, valved or unvalved masks, the reuse of masks, when to discard, as well as fake N 95 masks.

Valved masks have a one-way valve which is an exhalation valve [Figure 3]. This is very useful for the user as it provides comfort, prevents condensation inside the mask, misting

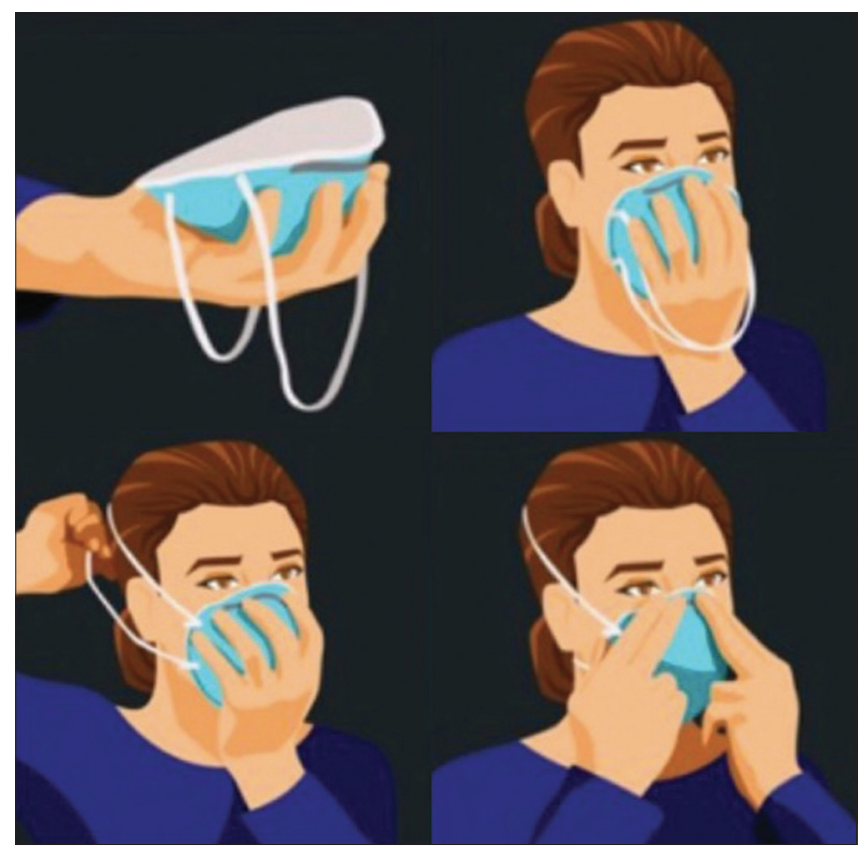

Figure 1: Demonstrates the steps in donning a mask of glasses, and helps the individual breathe easier. On the other hand, the unvalved traps heat and vapor from the mouth. The valved should not be used by a COVID positive as it will contaminate the surrounding. The user is safe, but the surroundings are not safe if the others are not wearing N95 masks.

Another important point is when to discard the N95 mask. If you see any tears or holes, if it does not form an effective seal to the face, it becomes wet or visibly dirty, contaminated with blood, body fluids, respiratory nasal secretion or after every aerosol generating encounter should be discarded. N95s can be reused. Air drying for 72 hours kills the virus, moist heat, chemical sterilization with $\mathrm{H} 2 \mathrm{O} 2$, Whatever is done should not affect the fit or compromise filtration capabilities. A simple way to reuse N95 is to keep three N95s, after each day put the N95 in the paper bag and then reuse after three days. ${ }^{[2]}$ Valve mask should be washed with water and soap. One should also be careful about fake N95s, this is a common issue that occurs when there is shortage. A recent study reveals $60 \%$ of N95 did not achieve required filtration, some only achieved 35\% filtration rather than $95 \% .{ }^{[3]}$ The N95 should be certified by the National Institute for Occupational Safety and Health (NIOSH).

An often asked question is which mask should be worn. Well, everybody should wear a mask and that is the law now. For non-healthcare workers, ideally, a surgical mask should be the choice, though this may not be possible as it is a disposable mask, multiple may be required in a day as they get moist easily and therefore may become unaffordable for the wearer. A 3-layer cotton mask is a useful alternative though not as effective. Its advantages are reusable and washable.

For healthcare workers, the ideal is an N95. If not available, surgical mask is an alternative. If a surgical mask is used it must be worn properly, with good hand hygiene and disposed of properly.

Face shield goggles: These are to be used to prevent contamination of mucus membranes of eyes, nose, mouth due to droplets generated by coughing, sneezing. They can help to prevent inadvertently touching the eye, nose, mouth with a contaminated hand and very useful during aerosol

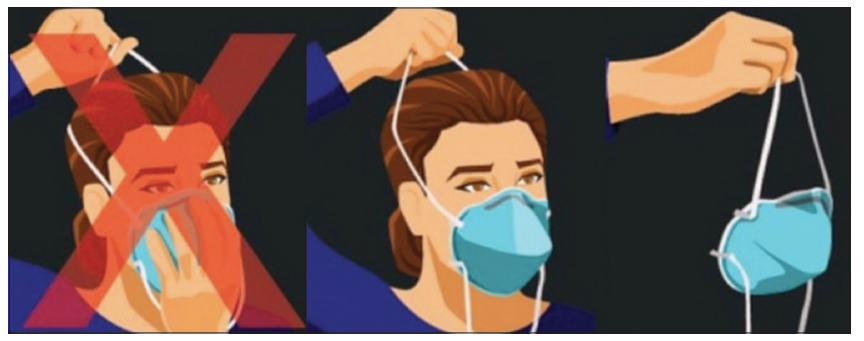

Figure 2: Doffing of N95 
generating procedures. Goggles should have a tight fit providing a good seal with the skin of face and surrounding regions. They should accommodate prescription glasses. Face shield should cover side of the face laterally, forehead superiorly as well as chin inferiorly. An anti-fog feature helps to improve clarity.

Gloves: These are useful in creating a barrier between the wearers hand and contaminated surfaces. It prevents a contaminated hand from directly touching the eyes, nose and mouth, thus breaking the contact chain. However cross contamination across surfaces may occur. In fact frequent hand washing is found to be superior. Nitrile gloves are recommended over latex as they are more chemical resistant to disinfectants especially chlorine. Allergic dermatitis may also occur to latex. Non-powdered are preferred over powdered.

Coveralls/gowns: These create a barrier, preventing exposure to contaminated droplets/secretions/body fluids. These are very useful when a healthcare worker needs to be within one meter of a COVID positive/suspect. Coveralls provide a 360-degree protection as they cover the entire body. Gowns are considered to be equally acceptable. There is a lack of a comparable evidence regarding the efficacy of overall and gowns. The gown should cover torso from the neck to the knee and arms till the wrist, as well as wrap around the back. These are fastened at the neck and the back. An apron can be worn over the gown. There are stringent standards for PPE quality in acting as an impervious barrier to biological and chemical hazards. These are monitored by many standards such as Sitara, Satara, DRDO, ISO-16603/ class 3 exposure pressure or equivalent.

Shoe covers: These are made of impermeable fabric to cover shoes providing personal protection preventing contamination of foot wear.

Head covers: Coverall do not require head covers as part of the coverall. When a gown is used or an aerosol generating procedure is done, a head cover is required. This should cover the head and back off the neck, all hair and hair extensions need to be included within the head cover.

Donning and doffing of coverall/gown. Similar to masks, the donning/doffing process is very important. Every donning and doffing process starts with hand hygiene. The order of donning and doffing vary between different institutions however the principles stay the same. The outer surface of the PPE is always considered contaminated, therefore the gown is removed after unfastening the ties and gown pulled away from the body at the level of the neck, shoulders, touching only the inside of gown. Once doffed the gown is turned inside out and folded and rolled up into a bundle and discarded in a waste container. Similarly, gloves are removed inside out, always sanitizing first and after.
Ideally doffing is done in front of a mirror or a colleague to ensure no mistakes are made, inadvertently the outer contaminated surface should not be touched.

\section{Surface Decontamination}

Surfaces should be divided into two types. Minimal hand contact surfaces such as floors and ceiling, these can be cleaned once a day. Frequent hand contact surfaces where there is frequent hand contact such as high touch surfaces, doorknobs, light switches, walls, keyboards, mouse etc. these should be cleaned more frequently, every four hours. There are different materials which can be used for sanitization of contact surfaces, 62 to $70 \%$ ethanol, 0.1 to $0.5 \%$ sodium hypochlorite, $2 \%$ glutaraldehyde, $0.5 \% \mathrm{H} 2 \mathrm{O} 2$. All these reduce the infectivity of the virus by a factor of 3 after minute of contact. It is important not to sweep rooms as this will cause aerosolization, wet mop should only be used. For equipment, monitors, keyboards, wet wipes should be used.

There may be aerolisation into examination rooms by a COVID positive patient, air exchanges are required to remove potentially infectious particles by circulating fresh air. The time required to change the air in the room with fresh air is important. This depends on the number of air exchanges. The greater the air exchanges, faster the room air is recirculated [Figure 4]. Generally most imaging departments have 6 air exchanges per hour. PETCT has 23/24 air exchanges per hour to remove isotopes. Therefore to is better to image covid patients in PETCT as the higher air exchanges ensure a shorter closure time of the CT examination room.

Equipment Cleaning: Surface decontamination of imaging equipment is very important especially after imaging COVID suspect/Covid positive patients.

Portable XRAY is the most commonly used imaging equipment in evaluation of COVID patients at the bedside. A dedicated portable $X$-ray machine should be placed in the COVID ward. This should not be moved out to avoid cross contamination. The machine should be covered by surgical drapes. Post imaging the drapes are discarded as infectious waste. The imaging detector is what comes in contact with the patient therefore needs the most attention. The detector is put inside two disposable bags, easy and practical are commonly available black garbage bags. The Radiographer dons PPE and takes the X-ray. Post X-ray the detector with double bag is removed. The outer bag is sanitized by the radiographer he then hands it to another radiographer waiting outside the COVID ward who takes the detector with inner bag, the outer bag is discarded as infectious waste by the radiographer in the COVID ward. The radiographer outside sanitizes the inner bag and then removes the detector processing, discarding the inner bag. The external surface of detector should also be sanitized. 
Sonography is often required for evaluation of deep venous thrombosis as well as for abdominal complaints. Initial recommendation for using sonography for lungs at point of care has not taken off. The process is quite time consuming requiring doing doffing of radiologist as well as sonography machine. There is a significant learning curve for radiologists to achieve competence. The information available doesn't seem to view what is available from a portable chest X-ray. Again a dedicated machine should be placed in the COVID ward. Preferably a hand held machine, if not cart type. The cart should be stripped of all paraphernalia such as printers gel bottles, gel sachets would be useful. The machine should be covered by drapes which post procedure are discarded. Post procedure the probes, cord and machine surfaces are wiped down with alcohol wipes. The probes may be covered in plastic.

CT scan has made a huge impact for TRIAGE and determining the severity of infection. Ideally, if two or more CT machines are available, then one should be dedicated for COVID. PETCT is utilized in the morning hours and is free in the afternoon onwards, it also has much higher air exchanges, so it is useful to utilize the PETCT for COVID CT. The CT table is covered with a disposable polythene sheet or soft cloth soaked in chlorine concentration of $2000 \mathrm{mg} / \mathrm{l}$, prior to scanning patient. Post procedure the table and gantry is cleaned with alcohol $60-75 \%$. The room is kept closed for air exchanges post procedure. The time required is based on the number of air exchange [Figure 4].

\section{Psychological aspects}

Imaging department staff suffer from psychological pressure especially the fear of contracting the disease at the work place, further carrying the disease home. Reassurance, education about safe practices and boosting morale are very important. Shorter working hours help a lot as they are away from home for shorter periods, encouraging frequent phone calls to their homes is very useful to reassure staff and home folks. Something many work practices did not encourage in the past! Personal calls. Family support helps boost the morale of staff a lot. If they are apprehensive about carrying the disease home they may be accommodated on the hospital premises or nearby hotels.

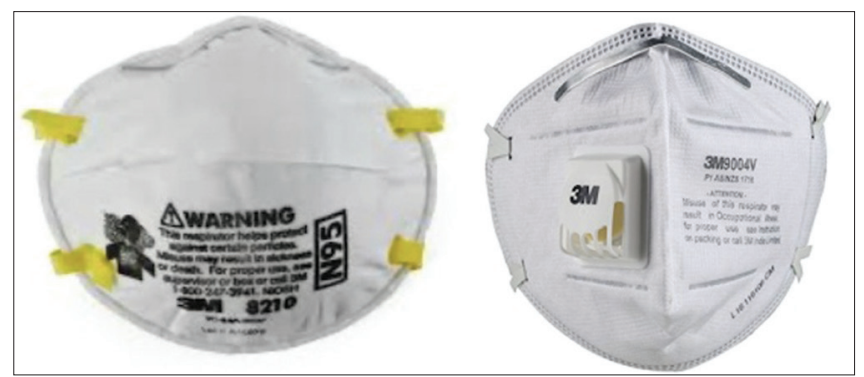

Figure 3: Unvalved and valved mask

\section{Economic aspects}

Due to social distancing and fear of stepping out, patients aren't visiting health care facilities, this has resulted in a $70 \%$ drop in imaging volumes. This is a problem for healthcare facilities, as imaging department works as a catalyst to generate work for health facilities. There is always a pressure to image! Which always works against social distancing. It is important to then cut costs, till the top line picks up again. One of the low hanging fruits is if you still print films. With the fear of fomites this is the best time to get out of this wasteful expenditure. Move to $\mathrm{CD}$, electronic transfer of images, cloud storage. This are

\section{Table B.1. Air changes/hour $(\mathrm{ACH})$ and time required for airborne-contaminant removal by efficiency *}

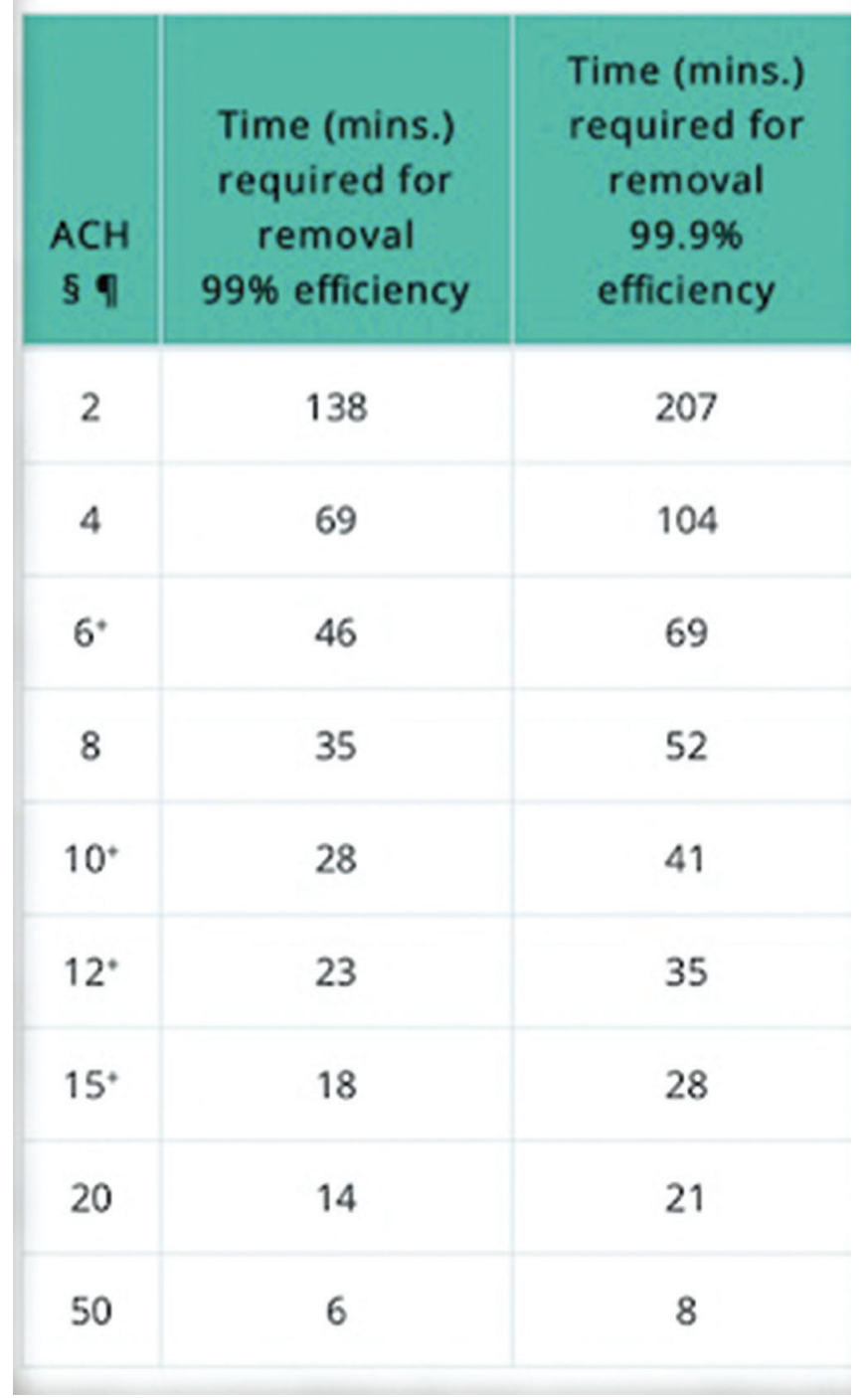

Figure 4: Time taken for Air exchanges to clear room 
all very reasonable and should help reduce the bottom line by $10-30 \%$. Other measures, reduce number of staff by automating appointments, patient queries, dictating and transcribing reports. Stop Referral fees. Imaging departments are going to feel the pinch for getting new equipment. Managements are not going to be keen to give high end equipment - toys for the boys- everything will need to be financially justified. An area of controversy is regarding the recent trend to have specialist and sub specialist imaging specialists. These are a significant expense on the professional side. Managements may be keen to curtail professional expenses and possibly look towards multimodality multi organ imaging specialists. On the flip side managements may be happy to outsource specialty reporting to imaging specialists who serve multiple centres/ institutions to cut professional costs.

With the COVID-19 pandemic showing no signs of letting up, we need to grapple with a new normal. Less work for sure, this may not be so bad, more time for hobbies, more time with family, more time to do what you always wanted to do. Hopefully this less work will allow us to move back from volume based practices, which we were all forced to get into, back into value based practices and thus bring healthcare back from being an industry with all its ills, to being a profession once again.

Finally COVID 19 appears to be supporting Darwin's theory of natural selection. Individuals with life style diseases are worse off with COVID 19, maybe a message for us to get fitter and for sure more hygienic!

Stay safe. Stay well.

\section{References}

1. Van Doremalen N, Morris DH, Holbrook MG, Gamble A, Williamson BN, Tamin A, et al. Aerosol and surface stability of SARS COV-2 as compared with SARS COV-1. N Engl J Med 2020;382:1564-7. doi: 10.1056/NEJM 2004973.

2. STANDARD OPERATING PROCEDURE (SOP) for extended use of N95 mask for personal safety of Health Care Workers (HCW) at AIIMS. Available from: https://www.aiims.edu/en/notices. [Last acessed on 2020 Apr 09].

3. Udwadia ZF, Raju RS. The N-95 mask: Invaluable ally in the battle against the COVID-19 pandemic. Lung India 2020;37:323-8.

This is an open access journal, and articles are distributed under the terms of the Creative Commons Attribution-NonCommercial-ShareAlike 4.0 License, which allows others to remix, tweak, and build upon the work non-commercially, as long as appropriate credit is given and the new creations are licensed under the identical terms.

\begin{tabular}{|l|l|}
\hline \multicolumn{2}{|c|}{ Access this article online } \\
\hline Quick Response Code: & \\
\hline & Website: \\
\hline
\end{tabular}

Cite this article as: Kohli A. COVID 19- Tips for getting back to work. Indian J Radiol Imaging 2020;30:105-10.

Received: 06-Jul-2020 Revision: 06-Jul-2020

Accepted: 06-Jul-2020
Published: 13-Jul-2020 